\title{
ON THE METRIC GEOMETRY OF THE PLANE N-LINE*
}

BY

\section{F. MORLEY}

The relations which $n$ lines of a plane exhibit, when considered in relation to the circular points, have not received systematic attention since the important memoirs by Clifford, On Miquel's theorem, Works, p. 51, and by Kantor, Wiener Berichte, vols. 76, 78 .

In what follows I shall apply certain notions which are fundamental in the geometric treatment of the theory of functions, and especially the notion of mapping. In the rational curves which alone are considered here, in place of expressing the Cartesian coördinates $X$ and $Y$ as real rational algebraic functions of a real parameter, we express $X+i Y$ or $x$ as a rational algebraic function of a parameter $t$, which it is convenient to make move on the unit circle. Thus uniformly the various numbers $t$ are of absolute value 1 ; for shortness they will be called turns. Curves then are here considered as maps of the unit circle ; and in general one equation, e. g.:

$$
x=a_{1}-2 a_{2} t+a_{3} t^{2},
$$

is sufficient. But of course such an equation carries with it the conjugate equation, which we write :

$$
y=b_{1}-2 b_{2} / t+b_{3} / t^{2}
$$

The coördinates $x, y$ or $X+i Y, X-i Y$ are called the circular coördinates of the point $x$.

\section{$\S 1$. The fixing of a line.}

To name a line it is enough to name the reflexion of the origin in that line. Thus by naming a point $x_{1}$ we name at the same time the line whose points are equidistant from 0 and from $x_{1}$.

To map the line on the unit circle we may write :

$$
x=\frac{x_{1} t_{1}}{t_{1}-t},
$$

\footnotetext{
* Presented to the Society February 24, 1900. Received for publication February 5, 1900.
}

Trans. Am. Math. Soc. 7 
where $t_{1}$ is an arbitrarily given turn. For in general an equation linear in both $x$ and $t$ maps the unit circle on another circle; but here $x$ is $\infty$ when $t$ is $t_{1}$, and the circle becomes a line.

Replace for a moment $t$ by any complex number $z$. When $z=\infty, x=0$; and when $z=0, x=x_{1}$. Now 0 and $\infty$ are inverse points of the unit circle; and it is an elementary theorem-but of considerable use - that inverse points map into inverse points. Here then 0 and $x_{1}$ are inverse points of the line.

The turn $t_{1}$ is at our disposal. We shall fix it by the condition :

$$
x_{1} t_{1}=y_{1}
$$

where $y_{1}$ is the conjugate of $x_{1}$.

The conjugate of (1) is

$$
y=\frac{y_{1} t}{t-t_{1}}
$$

whence the equation of the line in circular coördinates is

or

$$
x / x_{1}+y / y_{1}=1
$$

$$
x t_{1}+y=x_{1} t_{1}
$$

So when we are considering $n$ lines we take them as given by the reflexions of the origin $x_{1}, x_{2}, \cdots, x_{n}$ and take for their equations in circular coördinates :

where

$$
x t_{\alpha}+y=x_{\alpha} t_{\alpha}
$$

$$
x_{a} t_{a}=y_{a}
$$

$$
(a==1,2, \cdots, n)
$$

\section{§2. The center-circle and characteristic constants of an n-line.}

The two lines :

meet at the point :

$$
\begin{aligned}
& x t_{1}+y=x_{1} t_{1} \\
& x t_{2}+y=x_{2} t_{2}
\end{aligned}
$$

Consider three lines and let

$$
x_{12}=\frac{x_{1} t_{1}}{t_{1}-t_{2}}+\frac{x_{2} t_{2}}{t_{2}-t_{1}}
$$

$$
x=\frac{x_{1} t_{1}\left(t_{1}-t\right)}{\left(t_{1}-t_{2}\right)\left(t_{1}-t_{1}\right)}+\frac{x_{2} t_{2}\left(t_{2}-t\right)}{\left(t_{2}-t_{3}\right)\left(t_{2}-t_{1}\right)}+\frac{x_{3} t_{3}\left(t_{3}-t\right)}{\left(t_{3}-t_{1}\right)\left(t_{3}-t_{2}\right)} .
$$

This is of the form :

$$
x=a_{1}-a_{2} t
$$


and therefore is a circle whose center is $a_{1}$ and radius is $\left|a_{2}\right|$. But $x=x_{12}$ when $t=t_{3}$, and similarly the circle passes through the intersection of each two of the three lines. Thus the circle is the circumcircle of the 3-line, of which the circumcenter is

and the radius is $\left|a_{2}\right|$ where

$$
a_{1}=\sum \frac{x_{1} t_{1}^{2}}{\left(t_{1}-t_{2}\right)\left(t_{1}-t_{3}\right)},
$$

Consider 4-lines and let

$$
a_{2}=\sum \frac{x_{1} t_{1}}{\left(t_{1}-t_{2}\right)\left(t_{1}-t_{3}\right)} .
$$

$$
x=\sum \frac{x_{1} t_{1}^{2}\left(t_{1}-t\right)}{\left(t_{1}-t_{2}\right)\left(t_{1}-t_{3}\right)\left(t_{1}-t_{4}\right)} .
$$

Then $x$ moves on a circle; and, when $t=t_{4}, x$ is the circumcenter just found. But similarly when $t=t_{1}, t_{2}$, or $t_{3}, x$ is a circumcenter of a 3-line selected from the 4-line. Thus we have STEINER's theorem that the circumcenters of the four 3-lines selected from a 4-line lie on a circle. The center of SteINER's circle is

the radius is $\left|a_{2}\right|$ where

$$
a_{1}=\sum \frac{x_{1} t_{1}^{3}}{\left(t_{1}-t_{2}\right)\left(t_{1}-t_{3}\right)\left(t_{1}-t_{4}\right)}
$$

$$
a_{2}=\sum \frac{x_{1} t_{1}^{2}}{\left(t_{1}-t_{2}\right)\left(t_{1}-t_{3}\right)\left(t_{1}-t_{4}\right)} .
$$

But it is now clear that the reasoning admits of endless repetition. Thus from a 5 -line we have five 4-lines; the centers of their STEINER circles lie on a circle, a result given by Kantor (Über das vollständige Fünfseit, Wiener Berichte, vol. 78, p. 167). From a 6-line we have six 5-lines; the centers of their Kantor circles lie on a circle. Call this the center-circle of the 6-line. From a 7-line we have seven 6-lines; the centers of their center-circles lie on a circle. And so on ad infinitum.

For an n-line, when $n>2$, the center-circle is given by

where

$$
x=a_{1}-a_{2} t
$$

$$
\begin{aligned}
& a_{1}=\sum \frac{x_{1} t_{1}^{n-1}}{\left(t_{1}-t_{2}\right) \cdots\left(t_{1}-t_{n}\right)}, \\
& a_{2}=\sum \frac{x_{1} t_{1}^{n-2}}{\left(t_{1}-t_{2}\right) \cdots\left(t_{1}-t_{n}\right)} .
\end{aligned}
$$

Generally* we take, as characteristic constants of an n-line,

\footnotetext{
* For these characteristic constants of an $n$-line a double notation might be used, such as $a_{a}^{n}$, but it seems better in practice mentally to supply the number of lines we wish to consider, and to write merely $a_{a}$.
} 


$$
a_{\alpha}=\sum \frac{x_{1} t_{1}^{n-\alpha}}{\left(t_{1}-t_{2}\right) \cdots\left(t_{1}-t_{n}\right)}, \quad(a=1,2, \cdots, n) .
$$

The conjugate of $a_{a}$ is $b_{a}$ where

$$
\begin{aligned}
b_{\alpha} & =\sum \frac{y_{1} t_{1}^{a-n}}{\left(\frac{1}{t_{1}}-\frac{1}{t_{2}}\right) \cdots\left(\frac{1}{t_{1}}-\frac{1}{t_{n}}\right)} \\
& =(-)^{n-1} t_{1} t_{2} \cdots t_{n} \sum \frac{x_{1} t_{1} \cdot t_{1}^{n-2} \cdot t_{1}^{a-n}}{\left(t_{1}-t_{2}\right) \cdots\left(t_{1}-t_{n}\right)} \\
& =(-)^{n-1} t_{1} t_{2} \cdots t_{n} \cdot a_{n+1-a}
\end{aligned}
$$

that is, if one sets

$$
\begin{gathered}
s_{n}=t_{1} t_{2} \cdots t_{n}, \\
b_{a}=(-)^{n-1} s_{n} a_{n+1-a} .
\end{gathered}
$$

The $n$ equations (4) can be solved at once for $x_{1} \cdots x_{n}$. Thus

$$
x_{1}=a_{1}-a_{2} \sigma_{1}+a_{3} \sigma_{2}+\cdots+(-)^{n-1} a_{n} \sigma_{n-1},
$$

where $\sigma_{m}$ is the sum of products $m$ at a time of $t_{2}, t_{3}, \cdots, t_{n}$.

\section{§3. The node of an n-line.}

When we omit the line $x_{1}$ from the $n$-line we have an $(n-1)$-line whose constants are

$$
a_{1}-a_{2} t_{1}, \quad a_{2}-a_{3} t_{1}, \cdots, a_{n-1}-a_{n} t_{1} .
$$

When we omit two lines $x_{1}, x_{2}$ from the $n$-line we have an $(n-2)$-line whose constants are

And so on.

$$
\begin{gathered}
a_{1}-a_{2}\left(t_{1}+t_{.}\right)+a_{3} t_{1} t_{2}, \quad a_{2}-a_{3}\left(t_{1}+t_{2}\right)+a_{4} t_{1} t_{2}, \cdots . \\
a_{2} t_{1}, \quad a_{2}
\end{gathered}
$$

We consider now the equation :

$$
x=a_{1}-a_{2}\left(t_{1}+t_{2}\right)+a_{3} t_{1} t_{2} .
$$

When we replace $t_{2}$ by a variable $t$ we have the center-circle of the $(n-1)$-line obtained by omitting $t_{1}$. Thus if $t_{1}$ denote any assigned turn all the centercircles of the $(n-1)$-lines formed from an $n$-line are included in

$$
x=a_{1}-a_{2}\left(t_{1}+t\right)+a_{3} t_{1} t .
$$

We now prove that all these circles meet at a point. For the case $a_{2}=0$, the circles are all the same circle. 
For the case $a_{2} \neq 0$ suppose $x$ given. The equation

$$
a_{1}-x-a_{2}\left(z_{1}+z\right)+a_{3} z=0
$$

is that of a complex involution $I_{1}^{2}$, wherein to the unit circle on which $t$ moves corresponds another circle. The involution pairs off the circles in the plane and it is convenient to speak of the paired circles as partners. But circles can be their own partners, when, namely, they go either through the double points or about the double points (i. e., when the double points are inverse points of the circle). It is convenient to speak of these as circles of the involution, or as double circles.

When the unit circle is not a circle of the involution there are two cases: either the unit circle cuts its partner and then the intersections are a pair of points of the $I_{1}^{2}$, and the only pair on the unit circle, or the unit circle does not cut its partner and then the common inverse points of the two belong to $I_{1}^{2}$.

But when the unit circle is a circle of $I_{1}^{2}$, then there are $\infty$ pairs of $I_{1}^{2}$ on it forming a common projective involution.

All this is verified at once by means of the canonical form of $I_{1}^{2}$ :

$$
z+z^{\prime}=0 \text {. }
$$

When a relation involving turns, say

$$
f\left(t_{1}, t_{2} \cdots \mid a_{1}, a_{2}, \cdots\right)=0,
$$

is the same, save as to a factor, as the conjugate relation :

$$
f\left(\mathbf{1} / t_{1}, \mathbf{1}_{i} / t_{2}, \ldots \mid b_{1}, b_{2}, \cdots\right)=0,
$$

we shall say that the relation is self-conjugate.

Now in our case in order that when $z_{1}$ is any point $t_{1}$ on the unit circle the partner $z$ shall also be on the unit circle we require that the above equation (6) shall be self-conjugate. That is

and

$$
x=a_{1}-a_{2}\left(t_{1}+t\right)+a_{3} t_{1} t
$$

$$
y=b_{1}-b_{2}\left(1 / t_{1}+1 / t\right)+b_{3} / t_{1} t
$$

are to be not two equations determining $t_{1}$ and $t$ but merely one equation. And this is the case when

$$
x=a_{1}-a_{2} b_{3} / b_{2} ;
$$

for then the equation (6) is

$$
\frac{b_{3}}{b_{2}}-\left(t_{1}+t\right)+\frac{a_{3}}{a_{2}} t_{1} t=0
$$


which is manifestly self-conjugate. Whatever turn $t_{1}$ may be, a turn $t$ can be found satisfying this equation, and the corresponding value of $x$,

$$
x=a_{1}-a_{2} b_{3} / b_{2},
$$

is thus common to all the circles. Hence the theorem :

All the center-circles of the $(n-1)$-lines formed from an $n$-line meet at a point.

It is natural to consider the equation (6) in connection with the equation :

$$
x=a_{1}-2 a_{2} t+a_{3} t^{2},
$$

from which (6) is deduced by polarizing. This is the map-equation of a limaçon, the limaçon of the n-line.

What is the relation of the circles to the limaçon?

First. The $t_{1}$-circle and the limaçon have in common the point for which $t=t_{1}$.

Second. They touch at that point. For at $t_{1}$ one has

$$
\begin{aligned}
& D_{t} x=-a_{2}+a_{3} t_{1} \text { for the circle, } \\
& D_{t} x=-2 a_{2}+2 a_{3} t_{1} \text { for the limaçon; }
\end{aligned}
$$

whence the direction at the point is the same in both cases.

Third. The point where all the circles meet is the node of the limaçon. For when $x=a_{1}-a_{2} b_{3} / b_{2}$ we have

$$
\frac{b_{3}}{b_{2}}-2 t+\frac{a_{3}}{a_{2}} t^{2}=0
$$

so that we have the same point of the limaçon for two different values of $t$.

We may speak of this point as the node of the n-line.

A name is necessary for the system of circles, given by (6); that is, for the general system of circles passing through a point and having their centers on a circle.

I shall call them penosculants* of the limaçon :

$$
x=a_{1}-2 a_{2} t+a_{3} t^{2} .
$$

Generally when $x$ is a rational integral algebraic function of $t$, and the polynomial is polarized once, or more, the resulting curves may be termed penosculants.

Thus the limaçons of the $(n-1)$-lines formed from an $n$-line are included in

$$
x=a_{1}-a_{2}\left(t_{1}+2 t\right)+a_{3}\left(2 t_{1} t+t^{2}\right)-a_{4} t_{1} t^{2} ;
$$

* The name may serve to suggest the analogy as to formation with the theory of osculants of rational curves developed primarily by STuDy, Leipziger Berichte, 1886. The name oscuant was introduced by JoLLes in his Habilitationsschrift, Aachen, 1886. 
and these are first penosculants of the curve:

$$
x=a_{1}-3 a_{2} t+3 a_{3} t^{2}-a_{4} t^{3} .
$$

So the center-circles of all $(n-2)$-lines formed from the $n$-line are included in

$$
x=a_{1}-a_{2} s_{1}+a_{3} s_{2}-a_{4} s_{3},
$$

where $s_{a}$ is a sum of products of three turns, and these are second penosculants of the same curve.

We shall not examine this curve now, but shall rather seek to show by taking more familiar curves that there is a place for the theory of penosculants. But before leaving the method of the $a$ 's, we shall apply it to Clifford's theorem.

\section{§4. Clifford's chain. *}

From the point of view of this method Clifford's chainwise extension of MIQUEL's theorem is as follows:

The circumcircle of a three-line is :

$$
x=a_{1}-a_{2} t .
$$

The four such circles of a 4-line are included in

$$
x=a_{1}-a_{2}\left(t_{1}+t\right)+a_{3} t_{1} t
$$

and meet at the point (7),

$$
x=a_{1}-a_{2} b_{3} / b_{2}
$$

that is, since from (5) $b_{3} / b_{2}=a_{2} / a_{3}$ for a 4 -line, at the point

$$
x=a_{1}-a_{2}^{2} / a_{3},
$$

the two turns $t_{1}, t$ for this $x$ being the one $a_{2} / a_{3}$ and the other arbitrary.

The five such points of a 5 -line are included in

$$
x=a_{1}-a_{2} s_{1}+a_{3} s_{2}-a_{4} s_{3},
$$

where $s_{1}, s_{2}, s_{3}$ are the sums of products (one, two, three at a time) of three turns.

Now consider this complex involution, regarding $x$ as given. There is in an involution $I_{1}^{3}$ a neutral pair, that is a pair whose third element is arbitrary. Here the neutral pair is given by

$$
\left\{\begin{array}{l}
x=a_{1}-a_{2}\left(t_{1}+t\right)+a_{3} t_{1} t, \\
0=a_{2}-a_{3}\left(t_{1}+t\right)+a_{4} t_{1} t .
\end{array}\right.
$$

* Clifford's memoir (loc. eit. ) was published in 1870; KANTOR proved the same theorem in 1876, and subsequently published a second proof which is practically the same as CLIFFORD's. 
But for five lines the last equation is self-conjugate. Thus for any $x$ the neutral pair is on the unit circle; whatever turn $t_{1}$ may be, the turn $t$ can be found to satisfy the last equation; and the point $x$ is then on the circle :

$$
\left|\begin{array}{ll}
a_{1}-x & a_{2} \\
a_{2} & a_{3}
\end{array}\right|=t\left|\begin{array}{ll}
a_{2} & a_{3} \\
a_{3} & a_{4}
\end{array}\right|,
$$

found by eliminating $t_{1}+t$ and writing $t$ for $t_{1} t$. This then is Miquel's circle.

For six lines the two equations (9) become :

$$
\left\{\begin{array}{l}
x=a_{1}-a_{2} s_{1}+a_{3} s_{2}-a_{4} s_{3}, \\
0=a_{2}-a_{3} s_{1}+a_{4} s_{2}-a_{5} s_{3},
\end{array}\right.
$$

where the $s_{1}, s_{2}, s_{3}$ are for three turns. Now for six lines the last equation is self-conjugate. The equations determining the neutral pair of this involution $I_{1}^{3}$ are conjugate, namely :

$$
a_{2}-a_{3} s_{1}+a_{4} s_{2}=0, a_{3}-a_{4} s_{1}+a_{5} s_{2}=0,
$$

where $s_{\alpha}$ is a sum of products of two turns. Hence the neutral pair is on the unit circle. The equations of all MrQueL circles hold when

$$
\begin{aligned}
& x=a_{1}-a_{2} s_{1}+a_{3} s_{2}, \\
& 0=a_{2}-a_{3} s_{1}+a_{4} s_{2}, \\
& 0=a_{3}-a_{4} s_{1}+a_{5} s_{2}
\end{aligned}
$$

that is, all Miquel circles meet at the Clifford point :

$$
\left|\begin{array}{lll}
a_{1}-x & a_{2} & a_{3} \\
a_{2} & a_{3} & a_{4} \\
a_{3} & a_{4} & a_{5}
\end{array}\right|=0
$$

For seven lines the equations (10) determining the Clifford point become :

$$
\left\{\begin{array}{l}
x=a_{1}-a_{2} s_{1}+a_{3} s_{2}-a_{4} s_{3} \\
0=a_{2}-a_{3} s_{1}+a_{4} s_{2}-a_{5} s_{3} \\
0=a_{3}-a_{4} s_{1}+a_{5} s_{2}-a_{6} s_{3}
\end{array}\right.
$$

where the last two are conjugate. Eliminating $s_{1}$ and $s_{2}$ we see that the seven Clifford points lie on the Clifford circle :

$$
\left|\begin{array}{lll}
a_{1}-x & a_{2} & a_{3} \\
a_{2} & a_{3} & a_{4} \\
a_{3} & a_{4} & a_{5}
\end{array}\right|=t\left|\begin{array}{lll}
a_{2} & a_{3} & a_{4} \\
a_{3} & a_{4} & a_{5} \\
a_{4} & a_{5} & a_{6}
\end{array}\right|
$$


It is now clear that the argument is general and we can say: the Clifford point for $2 p$ lines is given by

$$
\left|\begin{array}{llll}
a_{1}-x & a_{2} & \cdots & a_{p} \\
a_{2} & a_{3} & \cdots & a_{p+1} \\
\vdots & & & \\
a_{p} & a_{p+1} & \cdots & a_{2 p-1}
\end{array}\right|=0
$$

and the equation of the Clifford circle for $2 p+1$ lines is given by

$$
\left|\begin{array}{cccc}
a_{1}-x & a_{2} & \cdots & a_{p} \\
a_{2} & a_{3} & \cdots & a_{p+1} \\
\vdots & & & \\
a_{p} & a_{p+1} & \cdots & a_{2 p-1}
\end{array}\right|=t\left|\begin{array}{cccc}
a_{2} & a_{3} & \cdots & a_{p+1} \\
a_{3} & a_{4} & \cdots & a_{p+2} \\
\vdots & & & \\
a_{p+1} & a_{p+2} & \cdots & a_{2 p}
\end{array}\right| \cdot
$$

We have thus the coördinates of Clifford's chain.

The CLIFFord circle ceases to exist if

$$
\left|\begin{array}{cccc}
a_{3} & a_{4} & \cdots & a_{p+1} \\
a_{4} & a_{5} & \cdots & a_{p+2} \\
\vdots & & & \\
a_{p+1} & a_{p+2} & \cdots & a_{2 p-1}
\end{array}\right|=0
$$

since then $s_{p}$ is a constant. But if no further conditions are imposed, we can solve the equations of the type (9) or (11) in the form :

and

$$
\begin{aligned}
& x=A_{0}+A_{1} s_{1}, \\
& x=A_{p}+A_{p-1} s_{p-1} \\
& y=B_{p}+B_{p-1} s_{1} / s_{p},
\end{aligned}
$$

whence on eliminating $s_{1}$ we have a line.

We turn now to the consideration of some penosculants.

\section{§5. Penosculants of the limaçon.}

The theory of five lines appears in its simplest form when considered in connection with the limaçon. Starting with this curve we write it

where $a$ and $\beta$ are real.

$$
x=2 a t+\beta t^{2},
$$

Here the center is the origin, the node is $-\beta$, the focus is $-a^{2} / \beta$. A penosculant (say $P_{1}$ ) is

$$
x=a\left(t_{1}+t\right)+\beta t_{1} t
$$


It appears from the (9) of the last section that the involution :

is of importance.*

$$
a+\beta\left(t_{1}+t\right)+a t_{1} t=0
$$

Eliminating $t_{1}+t$ we have

$$
\left|\begin{array}{rr}
x & a \\
a & -\beta
\end{array}\right|=t_{1} t\left|\begin{array}{rr}
a & -\beta \\
-\beta & a
\end{array}\right|,
$$

a circle with center at the focus, and passing through the node. We can call this the circle of inversion of the limaçon, since by an inversion in this circle the limaçon passes into itself.

Hence the point :

$$
\begin{aligned}
& x=a\left(t_{1}+t\right)+\beta t_{1} t, \\
& 0=a+\beta\left(t_{1}+t\right)+a t_{1} t,
\end{aligned}
$$

is the point where $P_{1}$ meets the circle of inversion (apart from the node).

Two penosculants $P_{1}$ and $P_{2}$ meet at

$$
x_{12}=a\left(t_{1}+t_{2}\right)+\beta t_{1} t_{2} .
$$

Consider now the expression:

$$
x=a\left(t_{1}+t_{2}+t\right)+\beta\left(t_{1} t_{2}+t_{1} t+t_{2} t\right)+a t_{1} t_{2} t .
$$

It is a circle with center $x_{12}$, and it passes through the points where $P_{1}$ and $P_{2}$ meet the circle of inversion. That is :

If two penosculants $P_{1}, P_{2}$ meet at $x_{12}$ and meet the circle of inversion at $m_{1}, m_{2}$, the node where all penosculants meet being disregarded, then a circle with center $x_{12}$ will pass through $m_{1}$ and $m_{2}$.

From the symmetry of (12) the three such circles for three penosculants meet at a point, namely:

or say

$$
x=a\left(t_{1}+t_{2}+t_{3}\right)+\beta\left(t_{1} t_{2}+t_{1} t_{3}+t_{2} t_{3}\right)+a t_{1} t_{2} t_{3},
$$

The conjugate equation is

$$
x=a s_{1}+\beta s_{2}+a s_{3},
$$

( 8 for 3 turns).

$$
y s_{3}=a s_{2}+\beta s_{1}+a .
$$

Multiply by $t_{4}$ and add. Then

$$
x+y s_{4}=a s_{1}+\beta s_{2}+a s_{3},
$$

( $s$ for 4 turns).

Hence: For four penosculants the four points lie on a line.

Naturally then five penosculants will give five lines and thus we get the Miquel figure as completed by KaNTOR.

* It is verified at once that points of the limaçon whose parameters are pairs of the involution are the ends of a focal chord. 
But, in fact, the theorem proved for four penosculants is the key to the theory of five lines.

From the standpoint of geometric drawing also this reverse view is the simplest. For the tedious determination of circles from three points is altogether avoided; the centers are given.

Still considering the limaçon :

$$
x=2 a t+\beta t^{2},
$$

we determine as follows the circle through the intersections (other than the node) of three penosculants.

We have for the intersection of two penosculants,

or

$$
x_{12}=a\left(t_{1}+t_{2}\right)+\beta t_{1} t_{2},
$$

$$
\beta x_{12}+a^{2}=\left(a+\beta t_{1}\right)\left(a+\beta t_{2}\right)=z_{1} z_{2} \text { say. }
$$

Hence the three points $x_{12}, x_{13}, x_{23}$ lie on the circle :

where

$$
\beta x+a^{2}=z_{1} z_{2} z_{3} / z
$$

$$
z=a+\beta t \text {. }
$$

In this mapping the focus $-a^{2} / \beta$ corresponds to $t=\infty$, the point $x=\infty$ to $t=-a / \beta$. The center of the circle or inverse of $x=\infty$ corresponds to the inverse of $-a / \beta$ as to the unit circle, or to $-\beta / a$, and is therefore

$$
\beta x+a^{2}=\frac{a}{a^{2}-\beta^{2}} z_{1} z_{2} z_{3} .
$$

The centers of the four such circles, for four penosculants taken by threes, lie on the circle :

$$
\beta x+a^{2}=\frac{a}{a^{2}-\beta^{2}} \frac{z_{1} z_{2} z_{3} z_{4}}{z} ;
$$

and the center of this circle is as before

$$
\beta x+a^{2}=\left(\frac{a}{a^{2}-\beta^{2}}\right)^{2} z_{1} z_{2} z_{3} z_{4} .
$$

We have then again a geometric chain proceeding ad infinitum. Thus the center-circle theorem proved in $\S 2$ for lines is true also for penosculants of a limaçon ; or explicitly :

Consider the circles whose centers lie on a given circle and which pass through a given point. Three of the circles have a circumcircle, or circle through their free intersections.

Four circles have four circumcircles; their centers lie on a circle. Call this the center-circle of the four. 
Five circles have five center-circles ; their centers lie on a circle. And so on. This theorem is the direct continuation of the theory of $\S 3$, and is parallel to that of $\S 2$. But there is also a parallel of $\S 3$.

For the center-circle of $n-1$ penosculant circles is

Now

$$
\beta x+a^{2}=\left(\frac{a}{a^{2}-\beta^{2}}\right)^{n-3} \frac{z_{1} z_{2} \cdots z_{n-1}}{z} .
$$

Hence if

$$
z z_{n}=(a+\beta t)\left(a+\beta t_{n}\right) \text {. }
$$

then

$$
z z_{n}=a^{2}-\beta^{2}
$$

$$
\beta+a\left(t+t_{n}\right)+\beta t t_{n}=0,
$$

a self-conjugate equation. Hence the center-circle passes through the point :

$$
\beta x+a^{2}=\frac{a^{n-3}}{\left(a^{2}-\beta^{2}\right)^{n-2}} z_{1} z_{2} \cdots z_{n} .
$$

That is, when we have $n$ circles whose centers lie on a circle and which pass through a point, if we take the center-circle of each set of $n-1$ circles, these $n$ center-circles meet at a point.

That Clifford's chain exists also for the penosculants of a limaçon is evident, for ClifFord's theorem is by inversion true when the system of lines is replaced by any system of circles through a point.

\section{§6. The quadratic involution arising from $2 p$ lines of a conic.}

When we invert the limaçon whose penosculants we have been considering into a conic the penosculants become the lines of the conic. We have theorems, which it is superfluous to state, in which the inverse of a focus of the conic as to a circle replaces the center of the circle. But some geometric statements will be facilitated by turning now to the conic, which we take to be an ellipse.

We write the ellipse

where $\mu$ is real.

$$
2 x=t+\mu^{2} / t,
$$

The center is the origin, the foci are $\pm \mu$, the axes are $\left|1 \pm \mu^{2}\right|$.

The tangent at $t_{1}, \mathrm{i}$. e., the line $t_{1}$ of the ellipse, is

$$
x=\frac{t_{1} t+\mu^{2}}{t_{1}+t} \text {. }
$$

For this is a line since $x=\infty$ when $t=-t_{1}$; the line passes through the point $t_{1}$ of the ellipse ; and it touches the ellipse at this point since when $t=t_{1}$ 


$$
\begin{aligned}
& D_{t} x=\frac{t_{1}^{2}-\mu^{2}}{4 t_{1}^{2}} \text { for the line, } \\
& D_{t} x=\frac{t_{1}^{2}-\mu^{2}}{2 t_{1}^{2}} \text { for the ellipse; }
\end{aligned}
$$

whence the direction of motion is the same in both cases.

From the mode of formation we may call this line a first penosculant of the ellipse.

The intersection of two such lines is the second penosculant, or penosculanto point :

We write (15) in the form :

$$
x_{12}=\frac{t_{1} t_{2}+\mu^{2}}{t_{1}+t_{2}}
$$

$$
\frac{x+\mu}{x-\mu}=\frac{t_{1}+\mu}{t_{1}-\mu} \frac{t+\mu}{t-\mu}
$$

or

$$
\frac{x+\mu}{x-\mu}=z_{1} z
$$

where

$$
z=\frac{t+\mu}{t-\mu}
$$

Then

$$
\frac{x+\mu}{x-\mu}=\frac{z_{1} z_{2} z_{3}}{z}
$$

is a circle; and, when $t=t_{3}, x=x_{12}$. Thus the circle is the circumcircle of the three lines of the ellipse.

When $t=-t_{4}$, then

$$
z=\frac{-t_{4}+\mu}{-t_{4}-\mu}=\frac{1}{z_{4}}
$$

Hence the four circumcircles of four lines of the ellipse meet at the point ;

$$
\frac{x+\mu}{x-\mu}=z_{1} z_{2} z_{3} z_{4} .
$$

For five lines the five such points lie on the circle :

$$
\frac{x+\mu}{x-\mu}=\frac{z_{1} z_{2} z_{3} z_{4} z_{5}}{z}
$$

the Miquel circle of the five lines. Evidently then :

The Clifford point of $2 p$ lines of an ellipse is

$$
\frac{x+\mu}{x-\mu}=z_{1} z_{2} \cdots z_{2 p} \text {. }
$$

The Clifford circle of $2 p-1$ lines is obtained by writing $1 / z$ for $z_{2 p}$. 
The inverse of the focus $\mu$ as to the circumcircle (16) is found as follows: One has the corresponding values:

$$
x=\mu, \quad z=0, \quad t=-\mu .
$$

The inverse of $-\mu$ as to the unit circle is $-1 / \mu$; therefore the inverse of $\mu$ as to the circumcircle is given by :

$$
\frac{x+\mu}{x-\mu}=\frac{1+\mu^{2}}{1-\mu^{2}} z_{1} z_{2} z_{3} .
$$

For four lines the four such points lie on the circle :

$$
\frac{x+\mu}{x-\mu}=\frac{1+\mu^{2}}{1-\mu^{2}} \frac{z_{2} z_{2} z_{3} z_{4}}{z}
$$

and the inverse of the focus $\mu$ as to this is as before

$$
\frac{x+\mu}{x-\mu}=\left(\frac{1+\mu^{2}}{1-\mu^{2}}\right)^{2} z_{1} z_{2} z_{3} z_{4} .
$$

Thus working with the one focus we have (for the first chain of $\S 5$ transferred to the ellipse) the series of points

$$
\frac{x+\mu}{x-\mu}=\left(\frac{1+\mu^{2}}{1-\mu^{2}}\right)^{n-2} \cdot z_{1} z_{2} \cdots z_{n} ;
$$

and in the same way for the other focus we have

$$
\frac{x^{\prime}+\mu}{x^{\prime}-\mu}=\left(\frac{1-\mu^{2}}{1+\mu^{2}}\right)^{n-2} \cdot z_{1} z_{2} \cdots z_{n}
$$

The inverse of the focus $\mu$ as to a ClIfFord circle is similarly, from (17),

$$
\frac{x+\mu}{x-\mu}=\frac{1+\mu^{2}}{1-\mu^{2}} z_{1} z_{2} \cdots z_{2 p-1}
$$

that of the other focus $-\mu$ is

$$
\frac{x+\mu}{x-\mu}=\frac{1-\mu^{2}}{1+\mu^{2}} z_{1} z_{2} \cdots z_{2 p-1} .
$$

If in the preceding process we take the foci alternately it is clear that, for any $2 p$ lines of the conic, we arrive at the Clifford point (17):

$$
\frac{x+\mu}{x-\mu}=z_{1} z_{2} \cdots z_{2 p}
$$

inasmuch as each factor $\left(1+\mu^{2}\right) /\left(1-\mu^{2}\right)$ introduced by using the positive focus $\mu$ is cancelled by the factor $\left(1-\mu^{2}\right) /\left(1+\mu^{2}\right)$ introduced by the other focus. It is clear also that we have entirely commutative geometric constructions; we 
arrive at this same point in whatever order we employ the foci, provided we use each $p-1$ times.

Thus for 6 lines we may take the inverses of $\mu$ as to all circumcircles; we get twenty points lying by fours on 15 circles-one for each 4-line; then the inverses of $-\mu$ as to these 15 circles, getting 15 points lying on 6 circles, 5 on each circle; then the inverses of $\mu$ as to these 6 circles, getting 6 points on a circle; and finally the inverse of $-\mu$ as to this circle. But the order in which the foci are taken is indifferent; the point arrived at is always :

$$
\frac{x+\mu}{x-\mu}=z_{1} z_{2} z_{3} z_{4} z_{5} z_{6}
$$

Consider the complex involution $I_{1}^{2}$ defined by

$$
\frac{x+\mu}{x-\mu} \cdot \frac{x^{\prime}+\mu}{x^{\prime}-\mu}=z_{1} z_{2} \cdots z_{2 p} \text {. }
$$

This involution has the following properties:

(i) Its center is the Clifford point of the $2 p$ lines.

(ii) The foci are a pair of the involution $I_{1}^{2}$.

(iii) The Clifford point of $2 q$ lines and that of the remaining $2(p-q)$ lines are a pair of $I_{1}^{2}$. The Clifford point of two lines means merely their intersection.

(iv) The Clifford circle of $2 q-1$ lines and that of the remaining lines are partners. The Clifford circle of a line is merely the line itself.

As to proof, (iii) is obtained by breaking up (18) into

$$
\begin{aligned}
& \frac{x+\mu}{x-\mu}=z_{1} z_{2} \cdots z_{2 q}, \\
& \frac{x^{\prime}+\mu}{x^{\prime}-\mu}=z_{2 q+1} \cdots z_{2 p} ;
\end{aligned}
$$

and (iv) follows from (iii) by considering that Clifford points lie on Clifford circles.

The circle of similitude of two circles is the locus of centers of involutions in which the two are partners. For any line through the center of an $I_{1}^{2}$ passes into a line through the center; hence the tangents from the center to a circle pass into the tangents from the center to the partner circle; whence by the principle of isogonality the partner circles subtend equal angles at the center. Hence :

Given $2 p$ lines of an ellipse, the circle of similitude of the Clifford circles of any 2q-1 lines and of the remaining lines passes through the Clifford point of all the lines. 
We might also consider higher involutions, for instance the $I_{2}^{3}$ :

$$
\frac{x+\mu}{x-\mu} \cdot \frac{x^{\prime}+\mu}{x^{\prime}-\mu} \cdot \frac{x^{\prime \prime}+\mu}{x^{\prime \prime}-\mu}=z_{1} z_{2} \cdots z_{n}:
$$

Thus for 6 lines of an ellipse we infer that all the triads of points obtained by dividing the 6 lines into three pairs belong to an $I_{2}^{3}$ of which the foci are the neutral pair.

\section{§ 7. Penosculants of a hypocycloid.}

The penosculants were defined in $\S 3$ for integral algebraic functions. But, as we have seen in the case of the ellipse, the notion may be advantageously used also when $x$ is a rational fractional algebraic function of $t$. We shall take an example of this, which is the key to an interesting generalization.

We consider the hypocycloid

The equation

$$
(n-1) x=n t-1 / t^{n} \text {. }
$$

$$
(n-1) x=t_{1}+(n-1) t-1 / t_{1} t^{n-1}
$$

defines a first penosculant $P$. It is a hypocycloid touching the given one at $t_{1}$. It has cusps when

or when

$$
D_{t} x=0
$$

$$
t_{1} t^{n}+1=0
$$

or when

$$
(n-1) x=n t-1 / t^{n} .
$$

Thus the cusps of a first penosculant are on the given hypocycloid. *

A second penosculant is defined by

$$
(n-1) x=t_{1}+t_{2}+(n-2) t-1 / t_{1} t_{2} t^{n-2} .
$$

It is the first penosculant at $t_{1}$ of the first penosculant at $t_{2}$; or it is the first penosculant at $t_{2}$ of the first penosculant at $t_{1}$.

And so on till we reach the completely polarized equation :

$$
(n-1) x=s_{1}-1 / s_{n},
$$

( $s$ for $n$ turns);

when one of the $n t$ 's is variable this equation is the equation of a segment of a line or penosculant line, and when all $t$ 's are fixed it defines a penosculant point, in which meet all the penosculant lines formed from $n-1$ of the $n t$ 's.

An independent meaning of this point will be proved later. First we develop its more obvious properties.

(i) Take $2 n$ points of the curve; divide them into any two sets of $n$ each ; all the centroids of the penosculant points of such two complementary sets lie on a line. For if

\footnotetext{
* See a memoir in the American Journal of Mathematics, vol. 16, p. 196.
} 
and

$$
(n-1) x=t_{1}+\cdots+t_{n}-1 / t_{1} \cdots t_{n},
$$

then

$$
(n-1) x^{\prime}=t_{n+1}+\cdots+t_{2 n}-1 / t_{n+1} \cdots t_{2 n},
$$

$$
\left.(n-1)\left(x+x^{\prime}\right)=s_{1}+t+1 / t s_{2 n}, \quad \text { (s for } 2 n \text { turng }\right),
$$

where $-1 / t=t_{1} t_{2} \cdots t_{n}$. But this is the map-equation of a segment of a line; hence all the points $\left(x+x^{\prime}\right) / 2$ lie on a line. The line is a penosculant line of

$$
2(n-1) x=(2 n+1) t+1 / t^{2 n+1} .
$$

(ii) Take $n+1$ points of the curve; the penosculant points of the various $n$ points are included in

$$
\left.(n-1) x=s_{1}-t-t / s_{n+1}, \quad \text { (s for } n+1 \text { turns }\right) .
$$

That is, the $n+1$ penosculant points lie on a circle.

(iii) Taking $n+2$ points of the curve we have $n+2$ such circles; writing

we see that

$$
t=-t_{1} t_{2} \cdots t_{n+2},
$$

$$
x=t_{1}+t_{2}+\cdots+t_{n+2}+t_{1} t_{2} \cdots t_{n+2} .
$$

Hence the $n+2$ circles meet in the point :

$$
x=s_{1}+s_{n+2}, \quad \text { ( } s \text { for } n+2 \text { turns). }
$$

(iv) Taking $n+3$ points of the curve we have $n+3$ such points, included in

$$
x=s_{1}-t+s_{n+3} / t, \quad(s \text { for } n+3 \text { turns }) .
$$

This last is the map-equation of a segment; hence the $n+3$ points lie on a line.

Take $n+4$ points of the curve ; the $n+4$ lines are included in

$$
x=s_{1}-t-t^{\prime}+s_{n+4} / t t^{\prime},
$$

( $s$ for $n+4$ turns),

and are therefore tangents of a hypocycloid of the third class.

For $n+5$ points of the curve the hypocycloids are penosculants of an asteroid, or hypocycloid of the fourth class. And so on.

When we come to $2 n+2$ points of the curve, say $t_{1}, t_{2}, \ldots, t_{2 n+2}$, we have again a hypocycloid which is merely the original one displaced. And there is this remarkable reciprocity, that if we take any $n$ of the points $t_{1}, t_{2}, \cdots, t_{2 n+2}$ of this second curve and repeat the process, selecting always from the remaining points, we recover the original curve. This reciprocity, for the case $n=2$, was found in another way by KANTOR.*

\footnotetext{
* Die Tangentengeometrie an der Steiner'schen Hypocycloide, Wiener Berichte, vol. 78, p. 232.

Trans. Am. Math. Soc. 8
} 
In this case of $n=2$ the meaning of the penosculant point is evident. The equation $(20)$ is

$$
x=t_{1}+t_{2}-1 / t_{1} t_{2} .
$$

For a variable $t_{2}$ this is the tangent at $t_{1}$ (or rather that segment of the tangent which is within the curve). Hence the point is the intersection of the tangents at $t_{1}, t_{2}$.

How to attach a meaning to the penosculant point (20) in general may be inferred from the case $n=4$.

The line-equation of the hypocycloid

is

$$
3 x=4 t-1 / t^{4}
$$

$$
x t^{4}+y t=t^{5}+1 \text {. }
$$

Consider also the curve whose line-equation is

$$
x t^{3}+y=x_{0} t^{3}+y_{0}+a t^{2}+b t .
$$

The lines for which

$$
t^{5}+1=x_{0} t^{4}+y_{0} t+a t^{3}+b t^{2}
$$

are common tangents to the two curves. Hence for five turns with $s_{5}=-1$ if

$$
x_{0}=s_{1}, \quad y_{0}=-s_{4}, \quad a=-s_{2}, \quad b=s_{3},
$$

these being pairs of conjugates by virtue of the relation $s_{5}=-1$, a curve (25) is determined which touches the 4 lines $t_{1}, t_{2}, t_{3}, t_{4}$ of the hypocycloid.

The line-equation of the curve is

$$
\left(x-s_{1}\right) t^{3}+y+s_{4}=-s_{2} t^{2}+s_{3} t
$$

and its point-equation is

$$
3\left(x-s_{1}\right)=-2 s_{2} / t+s_{3} / t^{2} .
$$

The curve is therefore a cardioid, and its center is

the point sought.

$$
x=s_{1}=t_{1}+t_{2}+t_{3}+t_{4}-1 / t_{1} t_{2} t_{3} t_{4},
$$

Thus the penosculant point of 4 points of the curve

$$
3 x=4 t-1 / t^{4}
$$

is the center of the (unique) cardioid which touches the 4 tangents.

It will be noticed that we are dealing with curves of direction.* The hypocycloid assigns a definite direction to its tangents, and these four directed lines determine the cardioid. There would be $2^{3}$ cardioids touching four lines not directed.

\footnotetext{
* Laguerre, Comptes R endus, 1865.
} 
The use of this determination of the penosculant point lies in the following parallel. This memoir handles mainly the intersections of $n$ lines, that is, the lines are primarily taken 2 at a time. There is a specially simple case, namely, when the lines all touch a hypocycloid of class three. From this case we have passed now to other simple cases : when all the lines touch other hypocycloids. But this gives the key to the general case of any $n$ lines; if we (continuing our example) group the lines 4 at a time we are to consider the cardioids which touch the lines by fours. To the centers of these cardioids the general theorems of the present memoir will equally apply; thus for 5 directed lines the centers of the cardioids will lie on a circle, and so on. In fact the algebra will be the same, the meaning of certain constants alone being altered.

To see this, we observe that a tangent of a cardioid whose center is $x_{0}$ is

$$
x t^{3}+y=x_{0} t^{3}+y_{0}+a t^{2}+b t
$$

or, if $p$ be the distance from the origin to this line,

$$
x_{0} t^{3}+y_{0}+a t^{2}+b t=2 p t^{\frac{3}{2}} .
$$

Hence for four given tangents

$$
x_{0}=2\left|\begin{array}{cccc}
p_{1} t_{1}^{2} & t_{1}^{2} & t_{1} & 1 \\
. . & . . & . . & . . \\
. . & . . & . . & . . \\
p_{4} t_{4}^{\frac{3}{2}} & t_{4}^{2} & t_{4} & 1
\end{array}\right| \div\left|\begin{array}{cccc}
t_{1}^{3} & t_{1}^{2} & t_{1} & 1 \\
\cdots & . . & . . & . . \\
\cdots & . . & . . & . . \\
t_{4}^{3} & t_{4}^{2} & t_{4} & 1
\end{array}\right| .
$$

If then we write $x_{1}$ for $2 p_{1} t_{1}^{\frac{3}{2}}$ we have:

$$
x_{0}=\sum \frac{x_{1}}{\left(t_{1}-t_{2}\right)\left(t_{1}-t_{3}\right)\left(t_{1}-t_{4}\right)},
$$

an expression of the form used in $\S 2$ and in fact that there denoted, for a 4line, by $a_{3}$.

Evidently then in the argument based on the constants $a$ we can substitute the center of the cardioid touching four directed lines for the intersection of two lines. And so in general for the intersection of two lines we may substitute the center $x_{0}$ of a curve touching $n+1$ directed lines, the line-equation of this curve being

$$
x t^{n}+y=x_{0} t^{n}+y_{0}+a t^{n-1}+b t+a^{\prime} t^{n-2}+b^{\prime} t^{2}+\cdots .
$$

HAVERFORD COLLEGE,

January, 1900. 